\title{
MENTIR VRAI ? LA SEPTIÈME FONCTION DU LANGAGE DE LAURENT BINET
}

\author{
Petr KYLOUŠEK \\ Université Masaryk, Brno
}

\begin{abstract}
En): Laurent Binet's novel is a game of simulation and dissimulation by its very intrigue. Who killed Roland Barthes and why? The police investigation conducted by the commissioner Jacques Bayard and the semiologist Simon Herzog is gradually transformed into questions about the relationship between fiction and reality and the nature of language. Is it knowledge or a misunderstanding? What is its noetic, ethical and political scope? What powers does it confer? The historical events of 1980 and the references to important personalities in political and scholarly life form a realistic screen that fiction manipulates, according to narrative strategies impacted by revelations and denials, by invalidated assertions and uncertain certainties. The analysis will attempt to define the axiological positioning of the narrator in relation to his characters, his narration and the status of the literary discourse between poetics and rhetoric, between the locutory and the illocutory/perlocutory. The main theoretical supports: Ch. K. Ogden and I. A. Richards, K. Bühler, GROUPE $\mu$ and Lubomír Doležel.
\end{abstract}

Keywords (En): language functions ; Jakobson's communication model ; fictional status of narration ; Binet ; French literature

Mots-clés (Fr): fonctions du langage ; modèle communicationnel de Jacobson ; statut de la fiction ; Binet ; littérature française

Le roman de Laurent Binet est un jeu de simulation et de dissimulation par son intrigue même. Qui a tué Roland Barthes et pourquoi ? L'enquête policière que mènent le commissaire Jacques Bayard et le sémiologue Simon Herzog se transforme au fur et à mesure en questionnement sur la relation entre la fiction et la réalité et sur la nature du langage. Est-il une connaissance ou une méconnaissance ? Quelle est sa portée noétique, éthique et politique ? Quels pouvoirs confère-t-il ? Est-il un instrument de communication ou plutôt un instrument de domination? Les événements historiques de 1980 et les références aux personnalités importantes de la vie politique et universitaire forment un écran réaliste que la fiction manipule en fonction de la stratégie narrative du discours littéraire situé entre la poétique et la rhétorique, entre le locutoire et l'illocutoire/perlocutoire.

Le roman se présente, on le devine, à la fois comme récit et métarécit où le discours sémiologique est mis en acte au sein d'une narration qui sert de métadiscours. La connaissance de ce qui est et le questionnement sur la réalité sont saisis en processus, autrement dit le noétique s'implique dans l'éthique et le politique, la parole est agie et agit, l'aventure des personnages, dont la majorité écrasante sont des intellectuels ou en passe de le devenir, est jalonnée d'indications qui, aux yeux des oulipiens, pourraient passer pour contraintes suggérant la voie à suivre aux personnages principaux qui - tel Simon Herzog et Slimane - finissent par acquérir cette septième fonction du langage et qui est «plus une clé, ou une piste, qu'une méthode» et que Jakobson aurait 
"effectivement désignée sous le terme de «"fonction performative" ». Sauf que «"performatif", c'est une image » (BINET, $2015: 458$ ).

Pour démêler l'écheveau, prenons pour indice-contrainte, le propos de Michel Foucault: "Ceci n'est pas une pipe, comme dirait Magritte, haha! (BINET, 2015 : 64) La scène se situe dans un sauna gay et, en citant Magritte, Foucault désigne son entrejambe où est calée la tête d'un jeune homme. Frivolité mise à part, nous sommes renvoyés aux notions centrales de la linguistique et de la connaissance, à savoir au triangle d'Ogden-Richards qui identifie la réalité (chose), la langue (mot) et le contenu mental (sens) comme trois éléments ontologiques distincts. Les relations qui fonctionnent entre ceux-ci n'en révèlent pas moins les seuils ontologiques qui les séparent : le mot n'est pas la chose, ni la notion, et la notion n'est pas la réalité. Le tableau de Magritte va plus loin encore en mettant en évidence la double distance entre la chose, son image et l'expression verbale. Et la citation de Foucault multiplie de surcroît le jeu des interprétations en transformant le signifié magrittien en signifiant d'un nouveau signifié à la manière barthesienne des Mythologiques sans pour autant renoncer à sa propre prise de distance ironique qui, elle, dédouble, encore, le jeu des significations. Or, on est loin du compte, car Michel Foucault de la page 64 n'est pas un énonciateur empirique, mais bien un personnage de roman raconté à la troisième personne par un narrateur désinvolte à focalisation mobile et qui entretient une distance ironique à signification variable aussi bien dans sa relation aux personnages que dans sa relation à la narration.

Ce long commentaire n'a pour but que d'indiquer l'espace potentiel d'où surgissent la simulation et la dissimulation en tant que facteurs de la dynamique narrative. Car les discontinuités ontologiques qui s'introduisent dans les relations entre les trois éléments du triangle, multipliées, de surcroît, par leurs implications à des niveaux différents de l'organisation du texte romanesque, se prêtent à la manipulation de chacun d'eux. Si dans la conception de l'École de Liège le Logos (mot) devrait servir de médiateur entre l'Anthropos (contenu mental) et le Cosmos (chose) (GROUPE $\mu, 1990$ : 96), Binet s'acharne à montrer la puissance du couple Anthropos/Logos comme créateur de la réalité dans l'interaction communicationnelle. Je propose de suivre la simulation (à la fois au sens de reproduction artificielle d'un processus et au sens de production d'une apparence imitée ou trompeuse) et la dissimulation (au sens de masque, secret tu, d'intention non déclarée ou feinte) sur trois pistes : thématisation, intentionnalité, statut de la fiction.

\section{Thématisation}

La première apparition verbis expressis de simuler et dissimuler sert à caractériser l'émulation entre deux étudiants qui cherchent à obtenir les faveurs d'une fille. L'émulation est matérialisée (métaphore, simulation ?) par une partie de billard :

[...] le garçon le mieux habillé convoite la jeune fille qui convoite l'autre garçon, plus débraillé, les cheveux longs un peu sales, dont le détachement légèrement arrogant ne permet 
pas encore d'affirmer si lui aussi s'intéresse à la jeune fille et s'il simule une indifférence statutaire liée à sa condition de mâle dominant qui sait d'évidence que la fille lui revient de droit, ou s'il attend quelqu'un, une plus belle, plus rebelle, moins timide, plus conforme à son standing (les deux hypothèses n'étant évidemment pas incompatibles). (BINET, 2015 : 104)

[...] répétition du coup à vide (la phase qu'on appelle «limage », se dit Simon) avec force gestes saccadés et trop rapides évoquant à la fois l'enjeu érotique de la partie et le manque d'expérience des joueurs, suivie d'une frappe sèche dont la rapidité ne suffit pas à dissimuler la maladresse. (BINET, $2015:$ 105, souligné par nous)

Dans les deux cas, une tension s'installe entre le code comportemental, les gestes et les paroles qui masquent ou au contraire imitent la réalité ou plutôt le rapport de vérité à la réalité.

Masquer la réalité par autre chose, faire savoir à l'un pour taire à l'autre est une situation narrative fréquemment utilisée. C'est le cas du dîner chez les Sollers (BINET, 2015 : 152-163) où simulations et dissimulations abondent : joute de paroles doucereuses entre Julia Kristeva et Hélène Althusser, relation lesbienne cachée entre Kristeva et la diplomate chinoise, drague sournoise de l'amie de Lacan qui excite Bernard Henry-Lévy sous la table, altercation entre Kristeva et Todorov, en bulgare, afin que les autres ne comprennent pas. Ce sont aussi les masques, celui de Mitterrand (BINET, 2015 : 288), ou ceux du carnaval de Venise (BINET, 2015 : 373 sqq.). C'est aussi le sentiment d'imposture qui poursuit Althusser (BINET, 2015 : 177 sqq., 262) ou Simon (BINET, 2015 : 167).

La liste serait longue. À chaque fois les signes qui simulent ou dissimulent agissent dans une situation. C'est par exemple le scandale que le trio Kristeva, Sollers, Bernard Henry-Lévy déclenche à La Salpêtrière pour attirer l'attention et permettre à deux agents bulgares d'entrer dans la chambre de Barthes mourant (BINET, 2015 : 66 sqq.). Ou bien la scène où le protagoniste focalisé sur Simon Herzog

ne voit pas Kristeva ni le vieil homme [Jakobson] à la coiffure d'arbuste avec sa cravate en laine mais ils sont là, pourtant, et s'il pouvait les voir, s'ils n'étaient pas chacun positionnés à des endroits différents mais sensiblement dissimulés dans l'ombre des invités, il verrait qu'ils ont tous les deux les yeux braqués sur Slimane et il saurait qu'ils ont tous les deux intercepté le signe que Slimane a fait avec ses mains et il devinerait qu'ils ont tous les deux deviné que le signe s'adressait à Derrida, caché, lui aussi, derrière son cercle d'admirateurs. (BINET, $2015: 347$ )

La simulation et la dissimulation sont imbriquées, indissociables de la réalité, car le mensonge exige la vérité pour être cru :

Kristoff sait parfaitement comment fonctionne un bon mensonge : il doit être noyé dans un océan de vérité. Avouer à $90 \%$ permet de crédibiliser les $10 \%$ qu'on cherche à dissimuler, et d'autre part cela réduit les risques de se couper. On gagne du temps et on évite de s'embrouiller. Quand on ment, il faut mentir sur un point et un seul, et être parfaitement honnête pour tout le reste. (BINET, $2015: 149$ )

Or la non-vérité peut être inhérente à la situation de communication, imputable à nos univers mentaux respectifs (nos bibliothèques au sens d'Umberto Eco) qui nous permettent aussi bien de sémantiser l'univers que d'empêcher de comprendre 
l'autre, comme dans cet interrogatoire de Roland Barthes par le commissaire Bayard :

«Y avait-il de l'argent dans votre portefeuille ? » [...] « Monsieur Barthes? Possédiez-vous quelque chose de valeur sur vous ? Pensez-vous qu'on aurait pu vous dérober quelque chose ? » Le silence qui règne dans la pièce est rompu seulement par le souffle rauque de Barthes qui passe dans le tube du respirateur. De longues secondes s'écoulent encore. Lentement, Barthes fait non, puis il détourne la tête. (BINET, 2015 : 24)

Si pour Barthes, valeur désigne le document qu'on lui a volé, pour Bayard, il s'agit d'une valeur qui se chiffrerait en argent comptant. Le mot, prenant un sens différent pour chacun des deux, est la source de la dissimulation d'autant mieux qu'à ce moment de l'intrigue Barthes s'abstient de préciser.

Or la langue elle-même dissimule car elle peut prêter à incompréhension et à erreur. Mourant, Barthes finit par indiquer ses assassins et la piste à suivre : «Sophia! Elle sait...» (BINET, $2015: 68$ ). Or, ce n'est que plus tard (BINET, 2015 : 178-179) que les deux enquêteurs, Bayard et Simon, comprennent qu'il ne s'agit pas d'une femme, mais bien de la capitale, métonymie des services secrets bulgares, et du sigle L.C., alias Logi Consilium devenu Logos Club, société secrète dédiée aux joutes rhétoriques où la maîtrise de la septième fonction du langage serait convoitée en tant qu'instrument du pouvoir.

Dans la construction du récit à mystère qu'est La septième fonction du langage et où le moteur même de l'action est le déchiffrage des indices qui renvoient à la réalité tout en la dissimulant, les différents aspects de la simulation/dissimulation se ramènent aux éléments et aux relations du triangle Ogden-Richards. Un autre facteur s'y ajoute - l'intentionnalité.

\section{Intentionnalité}

Laurent Binet se plaît à développer le contentieux qui oppose la filière anglosaxonne - Chomsky, Austin, Searle - à la française, représentée notamment par Derrida. La question centrale - celle des actes de langage (locutoires, illocutoires, perlocutoires) - ne fait pas l'unanimité. En effet, au colloque d'Ithaca, à la Cornell University, Jacques Derrida problématise l'approche essentialiste de John Searle, par une longue démonstration phénoménologique qui vise l'intentionnalité de la communication :

Lorsque j'écris, est-ce que je sais vraiment ce que je veux écrire ? Est-ce que le texte ne se dévoile pas à lui-même au fur et à mesure qu'il se formule ? (Se dévoile-t-il jamais vraiment ?) (BINET, $2015: 336)$

La communication même est envisagée comme une simulation (itérabilité) des paroles des autres.

Pour être entendu, au moins partiellement, par notre interlocuteur, nous devons employer la même langue. Nous devons répéter (réitérer) des mots qui ont déjà été utilisés, sans quoi notre interlocuteur ne pourra pas les comprendre. Nous sommes donc toujours, fatalement, dans une forme de citation. Nous utilisons les mots de l'autre. Or, comme pour le téléphone 
arabe, il est plus que probable, il est inéluctable, qu'au fil des répétitions, nous employions les mots des autres, tous autant que nous sommes, dans un sens légèrement différent les uns des autres. (BINET, $2015: 339$ )

Or l'itérabilité ne garantit pas encore la communication parfaite. En effet, il y a non seulement des écarts sémantiques interindividuels, mais on peut encore mettre en doute la définition essentialiste du monde actuel (real life) qui serait distinct du sujet et exempt de l'influence des facteurs subjectivisants :

La possibilité du parasitage est déjà là, même dans ce que Sarl appelle "real life », cette « real life » dont il est si assuré, avec une confiance (presque, not quite) inimitable, de savoir ce qu'elle est, où elle commence et où elle finit ; comme si le sens de ces mots ("real life ») pouvait immédiatement faire l'unanimité, sans le moindre risque de parasitage, comme si la littérature, le théâtre, le mensonge, l'infidélité, l'hypocrisie, le malheur (infelicity), le parasitage, la simulation de la real life ne faisait pas partie de la real life ! (BINET, 2015 : 340)

Non seulement l'homme est incapable de maîtriser entièrement son intentionnalité et l'impact de ses paroles sur l'autre, mais son acte même met en mouvement la réalité en s'y impliquant. Ainsi la simulation fait partie de la réalité simulée et la réalité simulée est réalité. On pourrait en dire autant de la dissimulation et de la réalité dissimulée. C'est cette dynamique que le roman de Laurent Binet met en œuvre en posant et illustrant la problématique de la relation fiction/réalité.

\section{Fiction}

Le statut fictionnel est signalé de deux manières. D’une part, par la thématisation de l'acte de l'écriture au niveau du narrateur où la simulation de la référence, le monde actuel, est mise en évidence :

Le commissaire s'installe dans un café, prend une bière, allume une gitane et ouvre $L e$ Roland Barthes sans peine. (Quel café ? Les petits détails, c'est important pour restituer l'ambiance, n'est-ce pas ? Je le vois bien au Sorbon, le bar en face du Champo, le petit cinéma d'art et d'essai, au bout de la rue des Écoles, mais à vrai dire, je n'en sais rien, vous pouvez le mettre où vous voulez.) (BINET, $2015: 32$ )

Cette thématisation concerne non seulement la mise en situation, mais également la diégèse :

Cette histoire possède un point aveugle qui est aussi son point de départ: le déjeuner de Barthes avec Mitterrand. C'est la grande scène qui n'aura pas lieu. Mais elle a eu lieu pourtant... Jacques Bayard et Simon Herzog ne sauront jamais, n'ont jamais su ce qui s'était passé ce jour-là, ce qui s'était dit. (BINET, 2015 : 188-189)

D'autre part, la thématisation de la fiction et de son rapport à la réalité référentielle se rattache au personnage de Simon Herzog, sémiologue, qui n'arrête pas de se demander s'il est en train de vivre la réalité ou une fiction romanesque :

Simon se demande : «Qu'est-ce que je ferais si j'étais dans un roman ? » [...] Mais la vie n'est pas un roman, se dit-il [...]. (BINET, $2015: 172$ ) 
Simon réfléchit pendant qu'il recule : dans l'hypothèse où il serait vraiment un personnage de roman [...], qu'est-ce qu'il risquerait vraiment ? Un roman n'est pas un rêve : on peut mourir dans un roman. Ceci dit, normalement, on ne tue pas le personnage principal, sauf, éventuellement, à la fin de l'histoire. Mais si jamais c'était la fin de l'histoire, comment le saurait-il ? Comment savoir à quelle page de la vie on est ? (BINET, $2015: 382$ )

[...] Simon précise : «Comment tu sais que tu n'es pas dans un roman ? Comment tu sais que tu ne vis pas à l'intérieur d'une fiction? Comment tu sais que tu es réel ? »/

Bayard considère Simon avec une curiosité sincère et lui répond sur un ton d'indulgence : T'es con ou quoi ? Le réel, c'est ce qu'on vit, c'est tout. » (BINET, 2015 : 420421)

Autrement dit, on entre dans le propos derridien. Et cela d'autant mieux que la simulation et la dissimulation sont productrices de réalité. À Venise, Simon est attiré par le spectacle - simulation de la bataille de Lepante (BINET, 2015 : 390392). Observateur, d'abord, il finit par entrer dans une autre réalité : "Simon n'écoute pas vraiment. Il s'avance vers le quai, fasciné par ce ballet de galères contrefaites et de barques maquillées. »(BINET, 2015 : 390)

Mais déjà auparavant, à Bologne, l'acte d'amour entre lui et Bianca est entièrement rythmé par L'Anti-CEdipe de Gilles Deleuze et de Félix Guattari et leurs «machines désirantes » (BINET, 2015 : 241-246). Le texte se fait chair et la réalité simule la parole, tout comme le spectacle de Venise simule la réalité de la bataille historique.

La relation entre la réalité du monde actuel et la fiction peut être réversible :

Il entend Eco expliquer à Bayard : «Cela reviendrait à dire que le Petit Chaperon rouge n'est pas en mesure de concevoir un univers où il y a eu la rencontre de Yalta et où Reagan va succéder à Carter. [...] Bref, pour le Petit Chaperon rouge qui jugerait un monde possible où les loups ne parlent pas, le monde "actuel" serait le sien, celui où les loups parlent » (BINET, $2015: 247)$

"Que signifie reconnaître à la lecture d'un roman que ce qui s'y passe est plus "vrai” que ce qui se passe dans la vie réelle ? » (BINET, $2015: 248$ )

Si nous rapportons les paroles d'Umberto Eco à l'idée derridienne de la simulation de la real life et qui fait partie de la real life, nous entrons non seulement dans le questionnement à la fois sémiologique et existentiel de Simon Herzog, mais aussi dans la matière même de la septième fonction du langage, sa performativité.

\section{La septième fonction du langage}

En effet, l'intrigue du roman à mystère que l'enquête policière de Jacques Bayard et de Simon Herzog est censée dévoiler se transforme en aventure du pouvoir la parole. Le noétique entre dans l'éthique et le politique, la parole se fait acte, la communication sert à dominer l'espace publique : l'enjeu des élections présidentielles de 1980 et le duel Giscard d'Estaing - Mitterrand qui sont le point de départ cèdent aux joutes et à la rivalité des universitaires au colloque de la Cornell University à Ithaca et aux joutes rhétoriques du Logos Club, une sorte de maçonnerie où, selon l'explication d'Umberto Eco 
[...], il y a deux grands courants du Logos Club : les immanentistes, qui trouvent dans le plaisir de la joute oratoire une fin en soi, et les fonctionnalistes, qui considèrent la rhétorique comme un moyen pour arriver à ses fins. Le fonctionnalisme se divise lui-même en deux courants: les machiavéliens et les cicéroniens. Officiellement, les premiers cherchent simplement à persuader, et les seconds plutôt à convaincre, les seconds auraient donc des motivations plus morales, mais dans les faits la distinction est floue puisque pour les deux, il s'agit d'acquérir du pouvoir ou de le conserver, alors... (BINET, $2015: 223-224$ )

La performativité de la fonction se matérialise par la rhétorique, l'art de s'affirmer plus fort, plus convaincant que l'autre, afin de le manipuler ou de manipuler un tiers. Deux trajectoires se développent à travers le récit. La première est celle de Slimane, un gigolo qui possède l'enregistrement de la septième fonction et qui à force d'écouter son baladeur apprend la technique. C'est un personnage secret qui traverse tous les obstacles au bout de quelques mots auxquels le lecteur n'aura pas accès du fait de la dissimulation narrative du narrateur. La seconde est celle de Simon Herzog qui, passant de la connaissance à l'action, aura affaire à la politique. Il va sauver sa vie in extremis en convainquant l'homme qui devait le tuer à tourner l'arme contre le commanditaire.

Le mot est ainsi une arme, un instrument efficace pour qui sait s'en servir. La joute oratoire où Simon doit réussir pour accéder au grade de tribun du Logos Club et qui l'oppose à un politicien napolitain est truffée de pièges dès le début (BINET, 2015 : 395 sqq.). Le sujet du duel est Classico e Barocco. Face à son adversaire qui défend la thèse que le classicisme n'existe pas et obligé par là de défendre la position opposée, Simon n'hésite pas à rompre le fil au moment d'être mis en difficulté :

Il décide de clore lui-même la séquence sur Shakespeare, qu'il estime avoir correctement négociée, et condense toute son énergie mentale pour changer de sujet, pour détourner son adversaire de la piste métadiscursive que celui-ci a commencé à creuser et où, pour la première fois, Simon ne se sent pas en sécurité.

«Un mot encore : Sérenissime. »

Ce disant il oblige son adversaire à être dans la réaction et, interrompu dans la séquence rhétorique qu'il s'apprêtait à bâtir, de nouveau privé de l'initiative, l'Italien rétorque : «Repubblica è barocco! » À ce stade d'improvisation, Simon joue la montre et dit tout ce qui lui passe par la tête : [...] L'italien veut répondre, mais Simon lui coupe la parole : «Ah, c'est vrai, j'oubliais : le Classique n'existe pas ! En ce cas, de quoi parlons-nous depuis une demi-heure ?» Le public bloque sa respiration. L'adversaire encaisse l'uppercut. (BINET, $2015: 406-407)$

Pour vaincre, il a fallu dissimuler sa faiblesse derrière l'écran des paroles, tout comme il faut dissimuler sa force en d'autres occasions, comme celle de la joute entre Philippe Sollers et Umberto Eco qui adopte «un ethos ultra-modeste et un niveau de développement très sobre et minimaliste. [...] Rigueur et humilité pour mettre en lumière le désordre mental de son interlocuteur mégalo. » (BINET, 201 : 416)

Tout compte fait, le parcours des deux personnages - Slimane et Simon - est une simulation, au sens technique du terme, de ce qui pourrait être la mise en pratique de la septième fonction du langage : une simulation par la fiction d'une 
fonction fictionnelle. Toujours est-il que pour la rendre crédible, comme l'est le mensonge de Kristoff cité ci-dessus, il lui faut simuler la réalité, en l'occurrence celle du monde universitaire et politique. Là aussi la simulation et la dissimulation vont de pair, car les personnages «réels » se mêlent aux «surnuméraires », selon la terminologie d'Umberto Eco, et les « réels », simulés, dissimulent la fiction : en effet, l'«authentique» Julia Kristeva, serait-elle vraiment la fille du chef des services secrets bulgares? John Searle aurait-il été l'assassin de Jacques Derrida et se serait-il suicidé ensuite en se jetant dans un torrent. Et l'assassinat de Roland Barthes ? Et ce flamboyant professeur Morris Zapp qui intervient au colloque d'Ithaca, ne serait-il pas pure fiction importée du roman Changing Places de David Lodge qui, il est vrai, aurait modelé son personnage sur un de ses collègues?

Ce qui vaut pour les personnages et les événements vaut aussi pour les nombreuses citations tirées du Degré zéro de l'écriture, des Mythologies, des Fragments d'un discours amoureux, de $S / Z$, du Roland Barthes sans peine, etc. En effet, où tracer la limite entre une citation exacte et son développement ou son extension dans les dialogues?

Et que dire de ce narrateur, si crédible, à la troisième personne, omniscient, et qui à certains moments se matérialise en je en signalant ainsi la nature fictionnelle du texte (voir ci-dessus). Mais n'est-ce pas aussi une manière de renvoyer, par l'intermédiaire de la distance ironique entre le narrateur et le je de l'auteur impliqué, à l'auteur empirique ? Simuler la présence de Laurent Binet, alors qu'il est dissimulé partout ailleurs :

Roland Barthes habitait rue Servandoni, à côté de l'église Saint-Sulpice, à deux pas du jardin du Luxembourg. Je vais me garer là où, je suppose, Bayard a garé sa 504, devant l'entrée du numéro 11. Je vous épargne le copier-coller, désormais d'usage, de la notice Wikipédia: l'hôtel particulier dessiné par tel architecte italien pour le comte de tel évêque de Bretagne, etc. (BINET, $2015: 50-51$ )

\section{En guise de conclusion}

Il convient sans doute de débarrasser, ici, les notions de simulation et de dissimulation de la connotation négative de fausseté et de mensonge, de nonréalité, de jeu gratuit, etc. dont la littérature s'est rendue suspecte aux yeux des philosophes, logiciens, ou linguistes et esthètes qui suivent la voie de Bertrand Russell, Gottlob Frege, Kendall L. Walton, mais aussi de John L. Austin ou John Searle. Nous n'entendons pas, ici, entrer dans les débats concernant la relation entre le monde actuel et la fiction. Contentons-nous de rappeler l'argumentation de Lubomír Doležel (DOLEŽEL, 2003: passim) sur la fictionnalité qui selon nous implique non seulement la littérature, mais fait partie de notre expérience quotidienne du monde actuel.

Ce que nous avons tenté de montrer est un des facteurs inhérent à la nature du langage en tant qu'instrument qui sert à la fois à saisir le monde et à exprimer les contenus de la pensée et des sentiments. Or, la dynamique de la communication soutient non seulement la noétique, mais s'inscrit également dans des stratégies communicationnelles de prise de position et de pouvoir. Les seuils ontologiques 
qui existent entre les trois éléments constitutifs du triangle Ogden-Richards et qui ne nous permettent de communiquer qu'indirectement, par l'intermédiaire du langage, sont en effet des failles qui ménagent aussi des espaces de manœuvre pour la simulation et la dissimulation. On pourrait conclure par la métaphore sportive du professeur Morris Zapp :

[...] «tout décodage est un nouvel encodage ». Si bien qu'en gros, on ne peut jamais être sûr de rien, et surtout pas que deux interlocuteurs se comprennent. [...] « La conversation est en somme une partie de tennis qu'on joue avec une balle en pâte à modeler qui prend une forme nouvelle chaque fois qu'elle franchit le filet. » (BINET, $2015: 313$ )

Serait-ce à dire que se faire comprendre relèverait du miracle ? Restons-en à Roland Barthes qui selon le Umberto Eco du roman de Laurent Binet aurait refusé la voie kabbaliste de l'interprétation du texte en suivant l'esprit de saint Augustin : s'il est «impossible de dire si une interprétation est valable ni si c'est la meilleure, [...] il est possible de dire si le texte refuse une interprétation incompatible avec sa propre textualité. Ça veut dire qu'on peut pas raconter n'importe quoi, quand même. » (BINET, 2015 : 217-218).

Espérons que c'est aussi le cas de la présente approche de La Septième Fonction du langage.

\section{BIBLIOGRAPHIE}

BINET Laurent (2015), La Septième Fonction du langage, Paris, Grasset.

BÜHLER Karl (1934), Sprachtheorie : die Darstellungsfunktion der Sprache, Jena, Gustav Fisher.

BÜHLER Karl (2009), Théorie du langage: la fonction représentationnelle, Marseille, Agone.

DOLEŽEL Lubomír (2003), Heterocosmica: fikce a možné světy, Praha, Karolinum. GROUPE $\mu$ (1990), Rhétorique de la poésie, Paris, Seuil. 